\title{
Least squares solutions of linear inequality systems: a pedestrian approach
}

\author{
Luis Contesse ${ }^{1}$, Jean-Baptiste Hiriart-Urruty ${ }^{2}$, Jean-Paul Penot ${ }^{3}$
}

"De tous les principes qu'on peut proposer pour cet objet, je pense qu'il n'en est pas de plus général, de plus exact, ni d'une application plus facile que celui qui consiste à rendre minimum la somme des carrés des erreurs"

"Of all the principles that can be proposed, I think there is none more general, more exact, and more easy of application, than that which consists of minimizing the sum of the squares of the errors"

A.-M.Legendre, Nouvelles méthodes pour la détermination des orbites des comètes, Paris (1805).

Abstract. With the help of elementary results and techniques from Real Analysis and Optimization at the undergraduate level, we study least squares solutions of linear inequality systems. We prove existence of solutions in various ways, provide a characterization of solutions in terms of nonlinear systems, and illustrate the applicability of results as a mathematical tool for checking the consistency of a system of linear inequalities and for proving "theorems of alternative" like the one by GoRDAn. Since a linear equality is the conjunction of two linear inequalities, the proposed results cover and extend what is known in the classical context of least squares solutions of linear equality systems.

Keywords. Linear inequalities, least squares solutions, convex polyhedron, quadratic function, alternative theorem.

Mathematics Subject Classification. 90C25, 93E24, 52A40, 65K10.

\section{Introduction}

The linear least squares problem is a mathematical and computational problem of primary importance, which originally arose from the need to fit a linear mathematical model to given observations. This corresponds to multiple observations, represented by the rows of $A$ and $b$, on a vector $x$. The observations may be inconsistent, and in this case a "solution" is sought. In mathematical terms, the resulting problem is to "solve" an overdetermined linear system of equations: given a vector $b \in \mathbb{R}^{m}$ and a $m \times n$ matrix $A$, with $m \geqslant n$, knowing that the system $A x=b$ has no solution, we want to find a vector $\bar{x} \in \mathbb{R}^{n}$ such that $A \bar{x}$ is the "best" approximation to $b$. But it remains the essential: to

\footnotetext{
${ }^{1}$ Facultad de Ingenieria, Pontificia Universidad Catolica de Chile, Santiago, Chile. lcontess@ing.puc.cl

${ }^{2}$ Institut de Mathématiques, Université Paul Sabatier, Toulouse, France. jbhu@math.univ-toulouse.fr

${ }^{3}$ Laboratoire J.L.Lions, Université P. et M.Curie, Paris, France.

jean-paul.penot@lijll.math.upmc.fr
} 
define what is the "best" solution. A choice which can be motivated for various reasons, including numerical and statistical ones, is to look for $\bar{x}$ solving the following minimization problem:

$$
\text { Minimize } g(x)=\frac{1}{2}\|A x-b\|^{2} \text { over } \mathbb{R}^{n},
$$

where $\|$.$\| stands for the usual Euclidean norm in \mathbb{R}^{n}$. The coefficient $1 / 2$ is there just to avoid trailing 2 in calculations dealing with differential calculus on $g$, and squaring the Euclidean norm "smoothes" the considered objective function (which is not necessarily the case for an arbitrary other norm in $\mathbb{R}^{n}$ ). The terminology "least squares" speaks for itself: we want to have the sum of squares $\sum_{i=1}^{m}\left(a_{i}^{T} x-b_{i}\right)^{2}$, where the $a_{i}$ 's are rows of $A$ and $b_{i}$ the components of $b$, as small as possible. This is the classical situation, well-understood from the mathematical and numerical viewpoints, see for example the book [1]. We shall recall some basics facts about it in the next section, as an appetizer for the more general case of inequalities. Motivated by not only applications but also mathematics, we move from the realm of "bilateral analysis" to that of "unilateral analysis" , that is from that of mere linear equalities to that of linear inequalities. Consider therefore a system of linear inequalities in $\mathbb{R}^{n}$

$$
a_{i}^{T} x \leqslant b_{i} \text { for } i=1,2, \ldots, m
$$

where $a_{i} \in \mathbb{R}^{n}, b_{i} \in \mathbb{R}$. In a packed form, we write $(\mathcal{S})$ as $A x \leqslant b^{5}$. Suppose that $(\mathcal{S})$ is infeasible, that is to say there is no $x$ satisfying all the inequalities in $(\mathcal{S})$. We are interested in "solving" $(\mathcal{S})$ in the best way. For that, we propose to tackle the following problem:

$$
\text { Minimize } f(x)=\frac{1}{2}\left\|(A x-b)_{+}\right\|^{2}=\frac{1}{2} \sum_{i=1}^{m}\left(a_{i}^{T} x-b_{i}\right)_{+}^{2} \text { over } \mathbb{R}^{n}
$$

where $r_{+}$stands for the positive part of $r$, i.e., $r_{+}=\max (r, 0)$. Like in the equality case where the residual to the aimed equality $a_{i}^{T} x-b_{i}=0$ was $\left|a_{i}^{T} x-b_{i}\right|$, the residual to the aimed inequality $a_{i}^{T} x \leqslant b_{i}$ (or $\left.a_{i}^{T} x-b_{i} \leqslant 0\right)$ is $\left(a_{i}^{T} x-b_{i}\right)_{+}$. So, it is natural, for solutions of $(\mathcal{Q})$, to speak of least squares solutions to linear inequalities $(\mathcal{S})$. The situation is however more complicated than in the equality case, to begin with: has problem $(\mathcal{Q})$ solutions? No theorem in Analysis allows us to answer immediately. Could we characterize the solutions of $(\mathcal{Q})$ as in the equality case (where the solutions are characterized as solutions of a linear system)? We thereafter plan to get down to answering these questions. We do not forget that an equality $a_{i}^{T} x=b_{i}$ amounts to two inequalities $a_{i}^{T} x \leqslant b_{i}$ and $-a_{i}^{T} x \leqslant-b_{i}$, with $\left(a_{i}^{T} x-b_{i}\right)^{2}=\left(a_{i}^{T} x-b_{i}\right)_{+}^{2}+\left(-a_{i}^{T} x+b_{i}\right)_{+}^{2}$; hence our results should cover the classical case of least squares solutions of linear equalities. To the best of our knowledge, the least squares problems for linear inequalities were firstly considered by HAN in [4], in a research report somewhat difficult to obtain. We shall explain below what is contained there, at least concerning existence results. But, let us begin with some appetizers.

\footnotetext{
${ }^{4}$ a terminology coined by J.J.MorEAU (1923-2014) to explain the development of his thoughts from Bilateral analysis to One-sided analysis, or from Linear analysis to Convex analysis.

${ }^{5}$ Here and elsewhere the inequalities between vectors are meant componentwise.
} 


\section{Two appetizers}

2.1 A brief account on least squares solutions for equality systems. We recall here some basic facts on the existence, uniqueness and characterization of solutions in the classical case of equality systems. The objective function $g$ in $(\mathcal{P})$ can be developed as

$$
g(x)=\frac{1}{2} x^{T}\left(A^{T} A\right) x-x^{T} A^{T} b+\frac{1}{2}\|b\|^{2} .
$$

Hence, $g$ is a nice quadratic convex function on $\mathbb{R}^{n}$, whose gradient $\nabla g(x)$ at $x \in \mathbb{R}^{n}$ is expressed as follows

$$
\begin{aligned}
\nabla g(x) & =\left(A^{T} A\right) x-A^{T} b \\
& =A^{T}(A x-b) .
\end{aligned}
$$

Since $\bar{x}$ is a minimizer of $g$ on $\mathbb{R}^{n}$ if and only if $\nabla g(\bar{x})=0$, we have at least two ways of proving the existence of solutions in $(\mathcal{P})$.

- Existence of solutions in $(\mathcal{P})$. Firstly by using Linear algebra or Matrix analysis. Clearly, $(\mathcal{P})$ is equivalent to

$$
\text { Minimize } \frac{1}{2}\|v-b\|^{2} \text { over all } v \in \operatorname{Im}(A)
$$

whence $(\mathcal{P})$ is the problem of projecting $b$ on the vector subspace $\operatorname{Im}(A)$. Another way to look at this existence question is as follows. The $n \times n$ matrix $A^{T} A$ is positive semidefinite with:

$$
\operatorname{Ker}\left(A^{T} A\right)=\operatorname{Ker}(A) ; \operatorname{Im}\left(A^{T}\right)=\operatorname{Im}\left(A^{T} A\right) .
$$

Hence the equation $\nabla g(x)=0$, that is to say

$$
\left(A^{T} A\right) x=A^{T} b
$$

also called "normal equations", does have solutions.

Secondly, by using Analysis and Optimization. When $A^{T} A$ is positive definite, the continuous function $g$ enjoys the so-called coercivity condition on $\mathbb{R}^{n}$, that is to say

$$
g(x) \rightarrow+\infty \text { as }\|x\| \rightarrow+\infty .
$$

So, minimizing $g$ on $\mathbb{R}^{n}$ amounts to minimizing it on some closed ball $\overline{B(0, R)}$ for $R>0$ large enough. By WEIERSTRASs theorem, we therefore are assured that a minimizer of $g$ exists. When $A^{T} A$ is just positive semidefinite, one has to rely on the coercivity of $g$ on a supplementary vector space of $\operatorname{Ker}\left(A^{T} A\right)$. In a single shot, one could use the following interesting result on unconstrained minimization of quadratic functions on $\mathbb{R}^{n}$ :

Let $B \in \mathcal{S}_{n}(\mathbb{R}), v \in \mathbb{R}^{n}$, and

$$
q: x \in \mathbb{R}^{n} \mapsto q(x)=\frac{1}{2} x^{T} B x-v^{T} x .
$$


Then the next three statements are equivalent:

(i) The function $q$ is bounded from below on $\mathbb{R}^{n}$;

(ii) $B$ is positive semidefinite and $v \in \operatorname{Im} B$;

(iii) The problem of minimizing $q$ over $\mathbb{R}^{n}$ has a solution.

Here, $B=A^{T} A$ and, by definition, the quadratic function $g$ is nonnegative on $\mathbb{R}^{n}$.

- Uniqueness of solutions in $(\mathcal{P})$ Again here, we have two ways of answering the question of uniqueness. Equation (4) has a unique solution if and only if $A^{T} A$ is invertible, that is to say is positive definite. That happens exactly when $\operatorname{Ker}\left(A^{T} A\right)=\{0\}$. So, following the first part of $(3)$, a necessary and sufficient condition for the uniqueness of solutions in $(\mathcal{P})$ is

$$
\operatorname{Ker}(A)=\{0\} .
$$

Note that this condition concerns only the matrix $A$, it is independent of the vector $b$. In the case when this condition for uniqueness does not hold, the solution set of $(\mathcal{P})$ is an affine space of the form $\bar{x}_{0}+\operatorname{Ker}(A)$, where $\bar{x}_{0}$ is an arbitrary solution of $(\mathcal{P})$. Consequently, even when there are several solutions $\bar{x}$, the "residual vector" $A \bar{x}-b$ remains constant.

- Characterization of solutions in $(\mathcal{P})$ As explained before, the solutions of $(\mathcal{P})$ are characterized by equation (4) For the numerical solution of $(\mathcal{P})$, there is a whole bunch of techniques specifically tailored for it, see [1].

2.2 Least squares solutions for inequality systems: the first drawbacks. Consider problem $(\mathcal{Q})$.We show here what are the properties similar to those in $(\mathcal{P})$ and, with the help of examples, what is completely different for that problem.

- The objective function in $(\mathcal{Q})$. The objective function $f$ in $(\mathcal{Q})$ can be viewed as $\sum_{i=1}^{m}\left(r \circ h_{i}\right)$, where $h_{i}: \mathbb{R}^{n} \rightarrow \mathbb{R}$ is the affine function defined by $h_{i}(x)=a_{i}^{T} x-b_{i}$, and $r$ : $\mathbb{R} \rightarrow \mathbb{R}$ is the increasing differentiable convex function defined by $r(t)=1 / 2\left(t_{+}\right)^{2}$. So, $f$ is a differentiable convex function with gradient

$$
\nabla f(x)=\sum_{i=1}^{m}\left(a_{i}^{T} x-b_{i}\right)_{+} a_{i}=A^{T}(A x-b)_{+} .
$$

We therefore are immediately faced with the following problems: contrary to $g$, the objective function $f$ to be minimized is no more quadratic and, possibly, not coercive on $\mathbb{R}^{n}$; equation (6) is no more linear... So, the results and techniques used in the previous subsection do not apply at all here. The specific question of existence will be considered in detail in the next section.

- Characterization of solutions in $(\mathcal{Q})$. Since we deal with a differentiable convex function, $\bar{x}$ is a minimizer of $f$ on $\mathbb{R}^{n}$ if and only if $\nabla f(\bar{x})=0$. So, the solutions in $(\mathcal{Q})$ are characterized by

$$
A^{T}(A x-b)_{+}=0,
$$

which we can also call normal equations. Unfortunately, due to the presence of the $(.)_{+}$ operation, the parts involving $x$ and $b$ cannot be separated like in (4). Note that $f$ is of class $\mathcal{C}^{1}$ on $\mathbb{R}^{n}$ but not of class $\mathcal{C}^{2}$. The gradient of $f$ is however LIPSCHITZ on $\mathbb{R}^{n}$,

$$
\|\nabla f(x)-\nabla f(y)\| \leqslant K\|x-y\| \text { for all } x, y \text { in } \mathbb{R}^{n},
$$


where a possible LiPsCHitz constant $K$ is, for example, $\|A\|^{2}$ for some ad hoc matricial norm $\|\cdot\|$.

- Some examples.

Example 2.2.1. Let $n=2, m=3$,

$$
A=\left[\begin{array}{cc}
1 & 0 \\
-1 & 0 \\
0 & 1
\end{array}\right], b=\left(\begin{array}{c}
-1 \\
-1 \\
1
\end{array}\right)
$$

Here, the least squares solutions for the inequality system $A x \leqslant b$ is the half-line $\{0\} \times$ $(-\infty, 1]$ of $\mathbb{R}^{2}$. We see in this example that $\operatorname{Ker}(A)=\{0\}$ but that the solution set in unbounded... Indeed, if $\bar{x}$ is a solution of $(\mathcal{Q})$, any element of $\bar{x}+\operatorname{Ker}(A)$ is also a solution of $(\mathcal{Q})$ (this is clear from the characterization $(7)$ ). So, to have $\operatorname{Ker}(A)=\{0\}$ is a necessary condition to have uniqueness of solutions in $(\mathcal{Q})$, but it is not sufficient. That is an important difference with the classical case of equalities.

Example 2.2.2. Let $n=2, m=4$,

$$
A=\left[\begin{array}{cc}
1 & 0 \\
-1 & 0 \\
0 & -1 \\
0 & 1
\end{array}\right], b=\left(\begin{array}{l}
-1 \\
-1 \\
-1 \\
-1
\end{array}\right)
$$

Here there is only one solution to the least squares problem for the inequality system $A x \leqslant b$, it is the origin $(0,0)$.

Example 2.2.3. Suppose that the vector inequality $A x \leqslant b$ is consistent, that is to say, there are points in $\mathbb{R}^{n}$ satisfying

$$
a_{i}^{T} x \leqslant b_{i} \text { for } i=1,2, \ldots, m
$$

a situation that could happen more likely than in the case of equalties. Then, clearly, the set of points satisfying (9) is the solution set of the least squares problem for $A x \leqslant b$. So, and that is again a difference with the classical case of equalities, any closed convex polyhedron in $\mathbb{R}^{n}$ can be a solution set for a least squares problem with inequalities.

\section{Tackling the question of existence of solutions}

As explained at the beginning of Section 2.2, none of the usual results from Analysis or Linear algebra leads us to an existence result in problem $(\mathcal{Q})$. We have to adapt techniques to this specific problem, that is what we shall do in various ways. For each proof, we shall say at the beginning what are the mathematical tools to be used.

First proof. It uses elementary techniques from Analysis (like extracting converging subsequences from bounded sequences) and Linear algebra (like $V \cap V^{\perp}=\{0\}$ for a vector 
space). Since $f$ is bounded from below (by 0 for example), we can consider its lower bound $\bar{f}=\inf _{x \in \mathbb{R}^{n}} f(x)$ and a minimizing sequence, that is a sequence $\left(x_{k}\right)$ such that $\left(f\left(x_{k}\right)\right)$ converges to $\bar{f}$ as $k \rightarrow+\infty$. If $f\left(x_{k_{0}}\right)=0$ for some integer $k_{0}$, i.e. if $a_{i}^{T} x_{k_{0}}-b_{i} \leqslant 0$ for all $i=1, \ldots, m$, then we are done: $x_{k_{0}}$ is a solution of problem $(\mathcal{Q})$. If not, by taking a subsequence if necessary, noted $\left(x_{k}\right)$ again, we can suppose that the nonempty sets of indices

$$
I_{k}=\left\{i: a_{i}^{T} x_{k}-b_{i}>0\right\}
$$

do not depend on $k$. We therefore denote by $I$ this common set of indices (defined in (10)).

First case: $\left(x_{k}\right)$ is bounded. Then there exists a subsequence $\left(x_{k_{l}}\right)$ of $\left(x_{k}\right)$ converging to $\bar{x}$ as $l \rightarrow+\infty$. As a consequence,

$$
f\left(x_{k_{l}}\right) \rightarrow \bar{f} \text { and } f\left(x_{k_{l}}\right) \rightarrow f(\bar{x}) \text { as } l \rightarrow+\infty,
$$

thus $f(\bar{x})=\bar{f}$ and $\bar{x}$ is a solution to $(\mathcal{Q})$.

Second case: $\left(x_{k}\right)$ is not bounded. Again by extracting subsequences, still denoted $\left(x_{k}\right)$, we can suppose that:

$$
f\left(x_{k}\right) \rightarrow \bar{f},\left\|x_{k}\right\| \rightarrow+\infty \text { and } \frac{x_{k}}{\left\|x_{k}\right\|} \rightarrow u \text { (unit vector of } \mathbb{R}^{n} \text { ) as } k \rightarrow+\infty .
$$

We then have

$$
\begin{aligned}
\frac{1}{2} \sum_{i \in I}\left(a_{i}^{T} u\right)^{2} & =\lim _{k \rightarrow+\infty} \frac{1}{2} \sum_{i \in I}\left(a_{i}^{T} \frac{x_{k}}{\left\|x_{k}\right\|}-\frac{b_{i}}{\left\|x_{k}\right\|}\right)^{2} \\
& =\lim _{k \rightarrow+\infty} \frac{1}{2\left\|x_{k}\right\|^{2}} \sum_{i=1}^{m}\left(a_{i}^{T} x_{k}-b_{i}\right)_{+}^{2}=\lim _{k \rightarrow+\infty} \frac{f\left(x_{k}\right)}{\left\|x_{k}\right\|^{2}}=0 .
\end{aligned}
$$

Hence $a_{i}^{T} u=0$ for all $i \in I$. We set

$$
V=\left\{v \in \mathbb{R}^{n}: a_{i}^{T} v=0 \text { for all } i \in I\right\} .
$$

We now decompose $x_{k}$ as $x_{k}=y_{k}+z_{k}$, with $y_{k} \in V$ and $z_{k} \in V^{\perp}$. By definitions of $V$ and $V^{\perp}$, we have

$$
\begin{aligned}
a_{i}^{T} x_{k} & =a_{i}^{T} z_{k} \text { for all } i \in I \text { and all } k \\
f\left(z_{k}\right) & =f\left(x_{k}\right) \rightarrow \bar{f} \text { as } k \rightarrow+\infty
\end{aligned}
$$

Now, the sequence $\left(z_{k}\right) \subset V^{\perp}$ will play the role played by the sequence $\left(x_{k}\right)$ in the first part of the proof.

First possibility: $\left(z_{k}\right)$ is bounded. There then exists a subsequence $\left(z_{k_{l}}\right)$ of $\left(z_{k}\right)$ converging to $\bar{z}$ as $l \rightarrow+\infty$. As a consequence,

$$
f\left(z_{k_{l}}\right) \rightarrow \bar{f} \text { and } f\left(z_{k_{l}}\right) \rightarrow f(\bar{z}) \text { as } l \rightarrow+\infty,
$$

thus $f(\bar{z})=\bar{f}$. 
Second possibility: $\left(z_{k}\right)$ is not bounded. Again by extracting subsequences, still denoted $\left(z_{k}\right)$, we can suppose that:

$$
f\left(z_{k}\right) \rightarrow \bar{f},\left\|z_{k}\right\| \rightarrow+\infty \text { and } \frac{z_{k}}{\left\|x_{k}\right\|} \rightarrow w\left(\text { unit vector of } V^{\perp}\right) \text { as } k \rightarrow+\infty .
$$

We have used above the property that $V^{\perp}$ is closed. Mimicking what we did above for the sequence $\left(x_{k}\right)$, we observe that

$$
\begin{aligned}
\frac{1}{2} \sum_{i \in I}\left(a_{i}^{T} w\right)^{2} & =\lim _{k \rightarrow+\infty} \frac{1}{2} \sum_{i \in I}\left(a_{i}^{T} \frac{z_{k}}{\left\|z_{k}\right\|}-\frac{b_{i}}{\left\|z_{k}\right\|}\right)^{2} \\
& =\lim _{k \rightarrow+\infty} \frac{1}{2\left\|z_{k}\right\|^{2}} \sum_{i=1}^{m}\left(a_{i}^{T} z_{k}-b_{i}\right)_{+}^{2}=\lim _{k \rightarrow+\infty} \frac{f\left(z_{k}\right)}{\left\|z_{k}\right\|^{2}}=0 .
\end{aligned}
$$

Hence $a_{i}^{T} w=0$ for all $i \in I$, that is: $w \in V$. But, since $V \cap V^{\perp}=\{0\}$, the unit vector $w$ cannot belong to both $V$ and $V^{\perp}$. So, this second possibility actually does not occur, the first one is in force and we are done.

Second proof. This proof is of a more advanced level, as it requires to know some Analysis on convex sets, like the theorem asserting the existence and uniqueness of the projection on a closed convex set. Let $K$ denote the closed convex cone of $\mathbb{R}^{m}$ consisting of vectors $u=\left(u_{1}, \ldots, u_{m}\right)$ whose components are all nonnegative. In the same manner, $-K$ is the closed convex cone of $\mathbb{R}^{m}$ consisting of vectors $u=\left(u_{1}, \ldots, u_{m}\right)$ whose components are all nonpositive. Working with theses cones is fairly easy, especially concerning the projections on them and the distances to them. When $u=\left(u_{1}, \ldots, u_{m}\right) \in \mathbb{R}^{m}$, we denote by $u^{+}=\left(u_{1}^{+}, \ldots, u_{m}^{+}\right)$and $u^{-}=\left(u_{1}^{-}, \ldots, u_{m}^{-}\right)$the two vectors with the following components respectively:

$$
u_{i}^{+}=\max \left(u_{i}, 0\right) ; u_{i}^{-}=\min \left(u_{i}, 0\right){ }^{6}
$$

Thus, $u^{+} \in K, u^{-} \in-K, u=u^{+}+u^{-}$and the two vectors $u^{+}$and $u^{-}$are orthogonal. Actually, $u^{+}$is the orthogonal projection of $u$ on $K$, while $u^{-}$is the orthogonal projection of $u$ on $-K$. This helps to calculate the squares of the distances of $u$ to $-K$ and to $K$ :

$$
d^{2}(u,-K)=\sum_{i=1}^{m}\left(u_{i}^{+}\right)^{2} ; d^{2}(u, K)=\sum_{i=1}^{m}\left(u_{i}^{-}\right)^{2} .
$$

Our initial problem consisted in minimizing $f(x)=\frac{1}{2} d^{2}(A x-b,-K)$ over all the $x \in \mathbb{R}^{n}$ :

$$
\text { Minimize } f(x)=\frac{1}{2} d^{2}(A x-b,-K) \text { over } \mathbb{R}^{n} .
$$

According to the definition of the distance $d(.,-K)$ to $-K$, this can be transformed into

$$
\text { Minimize } \frac{1}{2}\|A x-b-u\|^{2} \text { over all } x \in \mathbb{R}^{n} \text { and } u \in-K \text {. }
$$

\footnotetext{
${ }^{6}$ Beware that $u_{i}^{-}$is nonpositive, it is not what is usually called the negative part of $u_{i}$. To ease the reading of symbols, we use indifferently the notations $u^{+}$and $u_{+}$.
} 
This problem is posed in $\mathbb{R}^{n} \times \mathbb{R}^{m}$, its optimal value is $\bar{f}\left(=\inf _{x \in \mathbb{R}^{n}} \frac{1}{2}\left\|(A x-b)_{+}\right\|^{2}\right)$. We even go a step further: problem $\left(\mathcal{Q}^{\prime}\right)$ can be transformed into the following one:

$$
\begin{aligned}
\text { Minimize } \frac{1}{2}\|y-b-u\|^{2} \text { over all } y & \in \operatorname{Im}(A) \text { and } u \in-K \\
\text { Minimize } \frac{1}{2}\|v-b\|^{2} \text { over all } v & \in \operatorname{Im}(A)+K .
\end{aligned}
$$

This final problem $\left(\mathcal{Q}^{\prime \prime}\right)$ is posed in $\mathbb{R}^{m}$, its optimal value is still $\bar{f}$. Before comparing the solutions of $(\mathcal{Q}),\left(\mathcal{Q}^{\prime}\right)$ and $\left(\mathcal{Q}^{\prime \prime}\right)$, let us treat the problem $\left(\mathcal{Q}^{\prime \prime}\right)$ completely. The constraint set $\operatorname{Im}(A)+K$, sum of a linear subspace and of a polyhedral closed convex cone is convex and closed ([5, Corollary 19.3.2]). Hence, $\left(\mathcal{Q}^{\prime \prime}\right)$ consists in projecting the vector $b$ onto $\operatorname{Im}(A)+K$; it therefore has one and only one solution $\bar{b} \in \operatorname{Im}(A)+K$. How to link the solutions of $(\mathcal{Q}),\left(\mathcal{Q}^{\prime}\right)$ and $\left(\mathcal{Q}^{\prime \prime}\right)$ ? The construction and structure of the problems $(\mathcal{Q}),\left(\mathcal{Q}^{\prime}\right)$ and $\left(\mathcal{Q}^{\prime \prime}\right)$ speak by themselves:

- If $\bar{x}$ solves $(\mathcal{Q})$, then $(A \bar{x}-b)_{-}$is the projection of $A \bar{x}-b$ onto $-K$ and:

$$
\begin{gathered}
\left(\bar{x},(A \bar{x}-b)_{-}\right) \text {solves }\left(\mathcal{Q}^{\prime}\right) \\
\bar{b}=b+(A \bar{x}-b)_{+}=A \bar{x}-(A \bar{x}-b)_{-} \operatorname{solves}\left(\mathcal{Q}^{\prime \prime}\right) .
\end{gathered}
$$

- If $\bar{b}$ solves $\left(\mathcal{Q}^{\prime \prime}\right)$, then $\bar{b}$ expressed as $A \bar{x}+\bar{u}$ for some $\bar{u} \in K$ (and some $\bar{x}$ in $\mathbb{R}^{n}$ ) provides a solution $\bar{x}$ of $(\mathcal{Q})$.

This way at looking at $(\mathcal{Q})$ shows two things: firstly, there are solutions to $(\mathcal{Q})$; secondly, the "residual vector" $(A \bar{x}-b)_{+}$remains the same for all the solutions $\bar{x}$ to $(\mathcal{Q})$. We had observed the same phenomenon in the equalities case (see Section 2.1).

Third proof. This third proof is more in the lines of Optimization, it is also more involved. It was actually the proof proposed by HAN in [4, Section 2]. The main ingredient of the proof is a strong result which states the following : if a quadratic function (convex or not) is bounded from below on a closed convex polyhedron, then this lower bound is attained (there exists a minimizer of the quadratic function on the polyhedron). This result dates back to FrANK and Wolfe (1956) ; see also in [5, Section 27] how it can be derived from general results on the minimum of a convex function. The idea is to associate with $(\mathcal{Q})$ another problem $(\widehat{\mathcal{Q}})$, quadratic this time, but posed in $\mathbb{R}^{n} \times \mathbb{R}^{m}$, with the help of so-called "slack" variables $z \in \mathbb{R}^{m}$. Let us therefore consider

$$
\text { Minimize }_{x, z} \frac{1}{2}\|z\|^{2} \text { subject to } A x+z \leqslant b .
$$

Note that $P=\{(x, z): A x+z \leqslant b\}$ is a closed convex polyhedron in $\mathbb{R}^{n} \times \mathbb{R}^{m}$, but due to the lack of term $\|x\|^{2}$ in the objective function, $(\widehat{\mathcal{Q}})$ is not the problem of projecting the origin onto $P$. However, with the help of FRANK and Wolfe theorem quoted above, $(\widehat{\mathcal{Q}})$ does have a solution $(\bar{x}, \bar{z})$; the strict convexity of the function $\|\cdot\|^{2}$ makes moreover that $\bar{z}$ is unique. Now, how to link the solutions of $(\mathcal{Q})$ and $(\widehat{\mathcal{Q}})$ ? The following relationships come easily:

- If $\bar{x}$ solves $(\mathcal{Q})$, let $\bar{z}=(A \bar{x}-b)_{+} ;$then $(\bar{x}, \bar{z})$ solves $(\widehat{\mathcal{Q}})$. 
- If $(\bar{x}, \bar{z})$ solves $(\widehat{\mathcal{Q}})$, then $\bar{z}=(A \bar{x}-b)_{+}$necessarily and $\bar{x}$ solves $(\mathcal{Q})$. Let us summarize the results about existence of solutions in $(\mathcal{Q})$.

Theorem 1. For any matrix $A$ and vector $b$, the least squares problem $(\mathcal{Q})$ has a solution $\bar{x} \in \mathbb{R}^{n}$. The minimal "residual" vector $\bar{z}=(A \bar{x}-b)_{+} \in \mathbb{R}^{m}$ is unique, and $x \in \mathbb{R}^{n}$ is a least squares solution if and only if $(A x-b)_{+}=\bar{z}$.

To end this section, let us add some words about uniqueness of solutions in $(\mathcal{Q})$ and numerical procedures to find one solution to $(\mathcal{Q})$.

- Uniqueness. Contrary to the least squares problems for equality systems where things were clear, the case of inequality systems is more difficult: we do not know of any condition involving $A$ and $b$ alone (or $A$ alone) which would be necessary and sufficient for uniqueness of solutions in $(\mathcal{Q})$. Anyway, most least squares problems in practice usually give rise to several solutions.

- Numerical solutions. From the very beginning, HAN ([4, section 3]) proposed a specific algorithm to solve $(\mathcal{Q})$ numerically. Variants of HAN's algorithm can be found in $[2],[6$, Part II] , [7]. However, there now are numerous algorithms to solve this unconstrained convex differentiable minimization problem $(\mathcal{Q})$.

\section{Some applications}

When $a_{i}$ is a unit vector, $\left(a_{i}^{T} x-b_{i}\right)_{+}^{2}$ is the square of the distance $d\left(x, S_{i}\right)$ from $x$ to the half-space $S_{i}=\left\{x: a_{i}^{T} x \leqslant b_{i}\right\}$. Trying to find a point in $\cap_{i=1}^{m} S_{i}$ is a frequent objective in problems coming from signal and image processing (see [3] for example). However it may happen that requirements $x \in S_{i}$ for all $i$ be inconsistent; in that case, minimize $\sum_{i=1}^{m} d^{2}\left(x, S_{i}\right)$ is a sensible alternative; this is exactly the least squares problem $(\mathcal{Q})$. In addition to those practical applications, we have in mind applications in mathematics: the existence theorem of Section 3 can be a mathematical tool in devising proofs of results which, a priori, have nothing to do with least squares problems. Let us illustrate that with two examples.

4.1 A feasibility problem. Let $a_{1}, \ldots, a_{m}$ be $m$ vectors in $\mathbb{R}^{n}$, and let $b \in \mathbb{R}^{m}$. We consider the system of linear inequalities

$$
a_{i}^{T} x \leqslant b_{i} \text { for } i=1,2, \ldots, m .
$$

Question: is this system consistent? In other words: does there exist any $x \in \mathbb{R}^{n}$ satisfying $(\mathcal{S})$ ? Not always, of course... We give here a sufficient condition for that, involving only the $a_{i}$ 's, whatever be the $b_{i}$ 's in the right-hand side. For that, we assume the following on the $a_{i}^{\prime} \mathrm{s}$ :

$$
\left(\sum_{i=1}^{m} \lambda_{i} a_{i}=0 \text { with } \lambda_{i} \geqslant 0 \text { for all } i=1, \ldots, m\right) \Rightarrow\left(\lambda_{i}=0 \text { for all } i=1, \ldots, m\right) .
$$


The proof is easy by using the results displayed above. If $A$ denotes the matrix with rows $a_{1}, \ldots, a_{m}$, the assumption $(\mathcal{H})$ says that

$$
\left(A^{T} u=0 \text { with } u \geqslant 0\right) \Rightarrow(u=0) .
$$

Hence the solutions of the optimality condition $(7), A^{T}(A x-b)_{+}=0$, necessarily satisfy $(A x-b)_{+}=0$. But there are $x$ satisfying $(7)$, they are precisely the least squares solutions for the inequality system $A x \leqslant b$. Whence we have proved there indeed are points $x$ satisfying $(A x-b)_{+}=0$, that is $(\mathcal{S})$. A stronger assumption than $(\mathcal{H})$ would be to require $m=n$ and the $n$ vectors $a_{1}, \ldots, a_{n}$ linearly independent. This new assumption remains invariant if we change $a_{i}$ into $-a_{i}$, while $(\mathcal{H})$ does not. Indeed, condition $(\mathcal{H})$ is more adapted to systems of linear inequalities than the linear independence condition.

4.2 Alternative theorems. A theorem of alternative (or transposition theorem) is a set of two statements such that each one is false when the other is true. More precisely, let $P$ and $Q$ be two logical propositions. They are said to form an alternative if one and only one of them is true:

$$
(P \Rightarrow \operatorname{not} Q) \text { and }(\text { not } P \Rightarrow Q)
$$

or, just as simply:

$$
(P \Leftrightarrow \operatorname{not} Q) \text { or }[(\operatorname{not} P \Leftrightarrow Q)] .
$$

In the whole spectrum of alternative theorems, we choose GORDAN's alternative theorem, maybe the oldest one with propositions involving linear inequalities (1873). Let $a_{1}, \ldots, a_{m}$ be $m$ vectors in $\mathbb{R}^{n}$; consider the two next statements:

$(P) \quad: \quad$ There exists $x \in \mathbb{R}^{n}$ such that $a_{i}^{T} x<0$ for all $i=1, \ldots, m$;

$(Q)$ : There are nonnegative $\lambda_{i}$ 's, not all zero, such that $\sum_{i=1}^{m} \lambda_{i} a_{i}=0$.

GORDAN's alternative theorem expresses that (not $P \Leftrightarrow Q$ ). As usually the case in such equivalences, one way is easy (and without much interest), the other way is more difficult (and is the interesting part). Here, the implication $(Q \Rightarrow$ not $P)$ is easy and does not offer much interest. The converse implication (not $P \Rightarrow Q$ ) is what requires more effort to prove. We shall do that by using the existence result for least squares solutions of linear inequality systems. Assume (not $P$ ). For any $\varepsilon>0$, the system of linear inequalities

$$
a_{i}^{T} x \leqslant-\varepsilon \text { for } i=1,2, \ldots, m
$$

has no solution. Never mind, we consider a solution $\bar{x}$ of this system in the least squares sense, which does exist (see Section 3). Then, according to the optimality condition (7),

$$
\sum_{i=1}^{m}\left(a_{i}^{T} \bar{x}+\varepsilon\right)_{+} a_{i}=0 .
$$


We set $\lambda_{i}=\left(a_{i}^{T} \bar{x}+\varepsilon\right)_{+}$. All the $\lambda_{i}{ }^{\prime}$ 's are nonnegative and at least one of them is positive. If not, that would mean that the system of inequalities (16) is consistent, which is not the case. Whence $(Q)$ has been proved.

\section{Conclusion}

In this note, we have shown how the least squares problems with inequality systems differ from the classical ones with equality systems, especially as the question of existence is concerned. Elementary techniques and results in mathematics have been used in the proofs of the existence of solutions. As for applications, besides practical ones in signal and image processing, the least squares approach for inequality systems can be used as a mathematical tool. We have illustrated that in solving a feasibility problem and by providing a very short proof of GoRDAN's alternative theorem.

\section{References}

[1] A.BJÖrcK, Numerical methods for least squares problems. SIAM Publications (1996).

[2] R.Bramley and B.Winnicka, Solving linear inequalities in a least squares sense. SIAM Journal on Scientific Computing 17, 275-286 (1996).

[3] P.L.Combettes, Inconsistent signal feasibility problems: least squares solutions in a product space. IEEE Transactions on Signal Processing, Vol. 42, $\mathrm{n}^{\circ} 11,2955-2966$ (1994).

[4] S.P.Han, Least-squares solution of linear inequalities. Tech. Rep. TR-2141, Mathematics Research Center, University of Wisconsin-Madison (1980).

[5] R.T.Rockafellar, Convex analysis. Princeton University Press (Second printing 1972).

[6] J.H.Spoonamore, Least squares methods for solving systems of inequalities with application to an assignment problem, Technical Report TM FF-93/03, USACERL (1992).

[7] K.YAng, New iterative methods for linear inequalities, Technical Report 90-06, University of Michigan (1990). 